\title{
A new representation of polygonal curves based on piecewise-linear functions with potential application in shape identification
}

\author{
VICTOR M JIMENEZ-FERNANDEZ*, ENRIQUE VALDES-ORTEGA, JOSE A MARTINEZ- \\ MELCHOR, HECTOR VAZQUEZ-LEAL, URIEL A FILOBELLO-NINO and \\ NORBERTO CARRILLO-RAMON
}

Facultad de Intrumentación Electrónica, Maestría en Ingeniería Electrónica y Computación, Universidad Veracruzana, C.P. 91000 Xalapa, Veracruz, Mexico

e-mail: vicjimenez@uv.mx

MS received 1 February 2018; revised 15 July 2018; accepted 26 August 2018; published online 19 January 2019

\begin{abstract}
In this paper, a new representation of polygonal curves is proposed. The motivation behind this proposal is to find a descriptive model with potential application in shape identification. In particular, our work addresses the problem of identifying the shape of a given polygonal curve from a set of different ones. In accordance with the proposed representation, a curve is originally described as a series of consecutive points whose connection by straight lines sketches its characteristic shape. In order to define a proper identification scheme, each series of points is mapped to a one-dimensional piecewise-linear function that assigns to each point its corresponding angle. Depending on the case under study, this assignation is performed by following two possible alternatives: (i) in the case where the shape to be identified exactly corresponds with one of the previously stored ones, a sorted sequence beginning from the minimum value of angle and ending with the maximum one is considered, and (ii) in the case where the shape to be identified is similar to one of the previously stored ones, the assignation is performed by following the sequence of points as they appear in the polygonal curve (without sorting). Under this scheme of representation, by a cyclic comparative process between the computed functions, after several steps it is decided whether the graph of any input piecewise-linear function matches the polygonal curve to be recognized within a certain tolerance. In particular, in the case of the identification of two equal shapes, this proposal exploits the well-known principle of similarity geometry, which allows a polygon to be recognized independently of scale, translation and rotation. In order to validate this representation, a comparative analysis between two different shape identification methods (Fourier descriptors and canonical representation) and the piecewise-linear proposal is performed.
\end{abstract}

Keywords. Polygonal curves; shape identification; piecewise linear; similarity geometry.

\section{Introduction}

In this paper a new proposal based on polygonal curves [1-8] is approached. Taking this into consideration, let us first start with the definition of polygonal curve, which will become one of the main terms of interest in this paper.

Definition: Let $P_{0}, P_{1}, \ldots, P_{n}$ be different points on a Euclidean space. A polygonal curve $\mathbf{C}$ is defined as the union of the segments $\overline{P_{0} P_{1}}, \overline{P_{1} P_{2}}, \ldots, \overline{P_{n-1} P_{n}}$ described as

$$
\mathbf{C}=\bigcup_{i=1}^{n} \overline{P_{i-1} P_{i}}
$$

where the $i$ th point is denoted as

*For correspondence

$$
P_{i}=\left(x_{i}, y_{i}\right), \quad \text { for } \quad \mathrm{i}=0,1,2, \ldots, \mathrm{n}
$$

where $(n+1)$ is the number of points.

Given two consecutive segments $\overline{P_{i-1} P_{i}}$ and $\overline{P_{i} P_{i+1}}$ along the polygonal curve $\mathbf{C}$, it is said that $P_{i}$ represents a breakpoint vertex of the curve.

\section{Problem statement}

Let us consider that we are interested in identifying a specific polygonal curve $\mathbf{C}_{i}$, for $i=1,2, \ldots, N$, within a set $\Lambda=\left\{\mathbf{C}_{1}, \mathbf{C}_{2}, \ldots, \mathbf{C}_{N}\right\}$ of previously stored ones. Moreover, let a set of points $\lambda_{i}=\left\{P_{0}, P_{1}, \ldots, P_{n}\right\}$, for $i=1,2, \ldots, N$, be the unique information that is available to define each curve.

In this context, our first problem is to find a proper model to represent the shapes of polygonal curves. In this regard, 


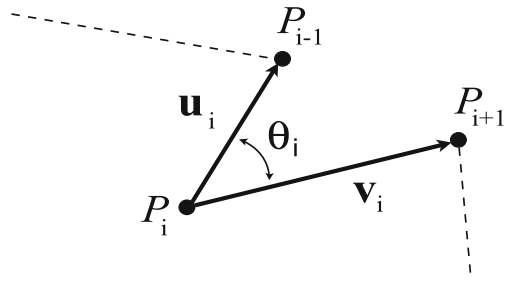

Figure 1. Arbitrary section of any polygonal curve.

although different types of representations are possible [9], a modified version of the so-called internal angle representation, referred to here as sorted angle representation, is proposed.

\section{Sorted angle representation and shape identification}

Before describing the sorted angle representation, it is important first to introduce the concept of breakpoint-angle. In this respect, consider figure 1, which represents an arbitrary section of any polygonal curve.

Figure 1 illustrates a particular breakpoint $P_{i}$ where two direction vectors, $\mathbf{u}_{i}$ and $\mathbf{v}_{i}$, are defined as follows:

$$
\begin{aligned}
\mathbf{u}_{i} & =\left(x_{i-1}-x_{i}\right)_{\mathbf{a}_{\mathbf{x}}}+\left(y_{i-1}-y_{i}\right)_{\vec{a}_{y}}, \\
\mathbf{v}_{i} & =\left(x_{i+1}-x_{i}\right)_{\mathbf{a}_{\mathbf{x}}}+\left(y_{i+1}-y_{i}\right)_{\vec{a}} y .
\end{aligned}
$$

The characteristic angle between such vectors $\theta_{i}$ is given by

$$
\cos \left(\theta_{i}\right)=\frac{\mathbf{u}_{i} \cdot \mathbf{v}_{i}}{\left|\mathbf{u}_{i}\right|\left|\mathbf{v}_{i}\right|} \quad \text { for } \quad \mathrm{i}=1,2, \ldots,(\mathrm{n}-1)
$$

or

$$
\begin{aligned}
\theta_{i} & =\cos ^{-1} \\
& \left(\frac{\left(x_{i+1}-x_{i}\right)\left(x_{i-1}-x_{i}\right)+\left(y_{i+1}-y_{i}\right)\left(y_{i-1}-y_{i}\right)}{\sqrt{\left(x_{i+1}-x_{i}\right)^{2}+\left(y_{i+1}-y_{i}\right)^{2}} \sqrt{\left(x_{i-1}-x_{i}\right)^{2}+\left(y_{i-1}-y_{i}\right)^{2}}}\right)
\end{aligned}
$$

with $0 \leq \theta_{i} \leq \pi$.

Our proposal is based on the assumption that a complete representation that implicitly preserves the shape of a particular polygonal curve can be achieved if we know the sorted sequence of the internal angles $\theta_{i}$ at each vertex (breakpoint-angles). For this reason, we refer to this type of description as sorted angle representation. In order to construct this representation, we firstly move along the polygonal curve and collect in a list the $(n-1)$-angles in the same order as they appear, starting from the first point $P_{0}$ and ending at the last one $P_{n}{ }^{1}$.

\footnotetext{
${ }^{1}$ It must be clarified that in the case of closed curves, two additional points must be taken into account for the angle computation: $P_{0}=P_{n}$ at the starting point, and $P_{n+1}=P_{1}$ at the ending point. In this case, the number of angles will be equal to the number of points $(n+1)$.
}

Then, the angles in the list are sorted into an ascending numerical order, and finally, from the sorted list, a onedimensional piecewise-linear function of the angles $\phi(j)$, for $j=1,2, \ldots,(n-1)$, is constructed ${ }^{2}$. This function relates two different sets of data: the characteristic angle $\theta_{i}$ and the $j$ th position using the explicit formulation $\phi(j)$ given by

$$
\phi(j)=a+b j+\sum_{i=1}^{n-3} c_{i}|j-i-1|
$$

where the form of the function $\phi(j)$ corresponds to the socalled canonical piecewise-linear Chua-Kang model [10-13], whose parameters can be determined as

$$
\begin{gathered}
c_{i}=\frac{\theta_{i+2}-2 \theta_{i+1}+\theta_{i}}{2} \text { for } \mathrm{i}=1,2, \ldots, \mathrm{n}-3, \\
a=2 \theta_{1}-\theta_{2}-\sum_{i=1}^{n-3}(i+1) c_{i}, \\
b=\frac{\left(\theta_{n-1}-\theta_{1}\right)-\left(\theta_{n-2}-\theta_{2}\right)}{2} .
\end{gathered}
$$

This step implies a mapping $\mathbf{C}_{i} \rightarrow \phi_{i}(j)$ that ensures the assignment of a unique angular function $\phi_{i}(j)$ for each list of points $\lambda_{i}$. More generally, the original set $\Lambda$ is mapped to a new set of functions $\Phi=\left\{\phi_{1}(j), \phi_{2}(j), \ldots, \phi_{N}(j)\right\}$.

Considering this, it can be stated that a specific polygonal curve $\mathbf{C}_{i}$ can be identified from a set $\Lambda$ when the following condition is reached:

$$
\min \left\{\epsilon_{1}, \epsilon_{2}, \ldots, \epsilon_{N}\right\}
$$

where $\epsilon_{i}$, for $i=1,2, \ldots, N$, is used as a deviation parameter to determine the difference between the input polygonal curve described by $\phi_{x}(j)$ and each one of the elements of $\lambda$ as follows: ${ }^{3}$

$$
\epsilon_{j}=\int_{1}^{(n-1)}\left|\phi_{i}(j)-\phi_{x}(j)\right| d j \text { for } \mathrm{j}=1,2, \ldots, \mathrm{N} .
$$

\subsection{Application example}

In order to illustrate how to apply the identification procedure described in the previous section, let us consider a set of three polygonal curves $\Lambda=\left\{\mathbf{C}_{1}, \mathbf{C}_{2}, \mathbf{C}_{3}\right\}$ where each curve is described by its corresponding list of points: $\lambda_{1}, \lambda_{2}$ and $\lambda_{3}$. As can be seen in figure 2 , the first curve describes the shape of a bird (figure 2a), the second one the shape of a rabbit (figure 2b) and the third the shape of a cat (figure 2c).

The lists of points that sketch the shapes of $\mathbf{C}_{1}, \mathbf{C}_{2}$ and $\mathbf{C}_{3}$ are given by

\footnotetext{
${ }^{2}$ In the case of closed polygonal curves, $j=1,2, \ldots,(n+1)$. ${ }^{3}$ It must be noted that in closed polygonal curves this parameter is expanded so that $\epsilon_{j}=\int_{1}^{(n+1)}\left|\phi_{i}(j)-\phi_{x}(j)\right| d j$.
} 


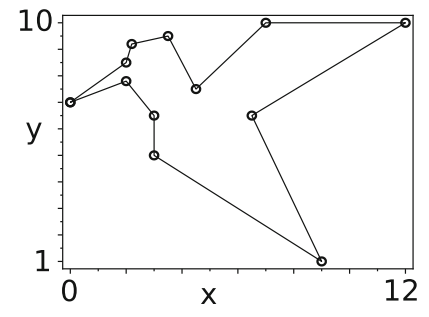

(a)

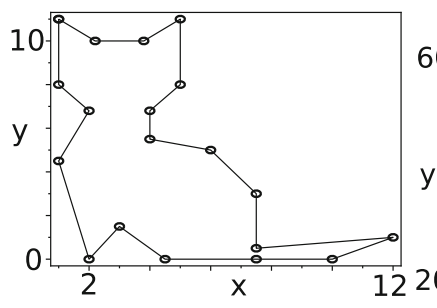

(c)

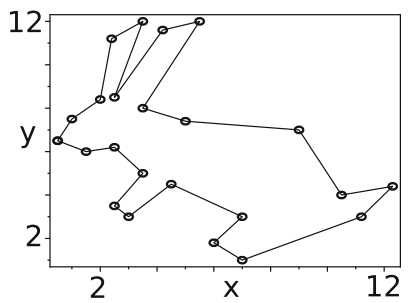

(b)

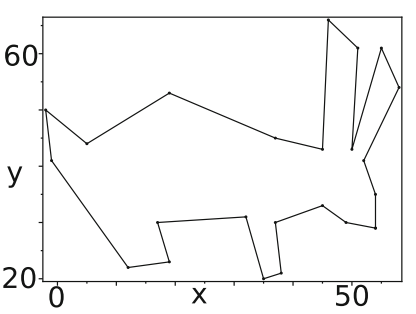

(d)
Figure 2. Polygonal curves of example 3.1. (a) $\mathbf{C}_{1}$ the bird, (b) $\mathbf{C}_{2}$ the rabbit and (c) $\mathbf{C}_{3}$ the cat. (d) $\mathbf{C}_{x}$ is the curve to be identified.

$$
\begin{aligned}
\lambda_{1}= & \{(0,7),(2,8.5),(2.2,9.2),(3.5,9.5),(4.5,7.5), \\
& (7,10),(12,10),(6.5,6.5),(9,1),(3,5),(3,6.5), \\
& (2,7.8),(0,7)\}
\end{aligned}
$$

$$
\begin{aligned}
\lambda_{2}= & (0.5,6.5),(1,7.5),(2,8.4),(2.4,11.2),(3.5,12), \\
& (2.5,8.5),(4.2,11.6),(5.5,12),(3.5,8),(5,7.4), \\
& (9,7),(10.5,4),(12.3,4.4),(11.2,3),(7,1), \\
& (6,1.8),(7,3),(4.5,4.5),(3,3),(2.5,3.5), \\
& (3.5,5),(2.5,6.2),(1.5,6),(0.5,6.5)\} \\
\lambda_{3}= & ((1,11),(2.2,10),(3.8,10),(5,11),(5,8), \\
& (4,6.8),(4,5.5),(6,5),(7.5,3),(7.5,0.5), \\
& (12,1),(10,0),(7.5,0),(4.5,0),(3,1.5), \\
& (2,0),(1,4.5),(2,6.8),(1,8),(1,11)\}
\end{aligned}
$$

Now, suppose we are interested in determining whether the shape of the curve $\mathbf{C}_{x}$, shown in (figure 2d) and described by $\lambda_{x}$, matches any of the previously known in $\Lambda$, where the list of points of $\mathbf{C}_{x}$ is given by

$$
\begin{aligned}
\lambda_{x}= & \{(54,29),(54,35),(52,41),(58,54),(55,61), \\
& (50,43),(51,61),(46,66),(45,43),(37,45), \\
& (19,53),(5,44),(-2,50),(-1,41),(12,22), \\
& (19,23),(17,30),(32,31),(35,20),(38,21), \\
& (37,30),(45,33),(49,30),(54,29)\}
\end{aligned}
$$

At first glance, in spite of being at a different scale and rotated, the similarity between the shape of $\mathbf{C}_{x}$ and $\mathbf{C}_{2}$ is clear; however, this visual intuition must be formally proven. In this sense, the procedure described in the previous section is applied. For this, we firstly compute the direction vectors $\left(\mathbf{u}_{i}, \mathbf{v}_{i}\right)$ for each polygonal curve $\left(\lambda_{1}, \lambda_{2}\right.$, $\lambda_{3}$ and $\lambda_{x}$ ). Then, from these vectors, a set of breakpointangles $\theta_{i}$ is also determined for each curve. After this, each one of these sets is sorted in ascending order in accordance with the values of its elements $\theta_{i}$. With this, based on Eqs. (6)-(9), we construct the corresponding piecewiselinear functions: $\phi_{1}(j), \phi_{2}(j), \phi_{3}(j)$ and $\phi_{x}(j)$, leading to the following results:

$$
\begin{aligned}
\phi_{1}(j)= & 84.92665746+.4945501050 j+19.24432214|j-2| \\
& -6.31583628|j-3|-10.45949470|j-4|-1.673224410|j-5| \\
& +5.488041050|j-6|-4.211387700|j-7|+3.279938850|j-8| \\
& -1.939262300|j-9|-3.523761150|j-10| \\
\phi_{2}(j)= & 16.80925485+15.29487567 j-11.97304814|j-2| \\
& +2.865759600|j-3|+13.90385572|j-4|-15.37389637|j-5| \\
& -.230147005|j-6|-1.236057995|j-7|+5.082084820|j-8| \\
& -4.291810800|j-9|-1.363155450|j-10|+1.234059000|j-11| \\
& +3.452183650|j-12|-3.376846700|j-13|+.580606900|j-14| \\
& +4.050912850|j-15|-5.098391450|j-16|+1.294871050|j-17| \\
& -1.133544550|j-18|+4.743926950|j-19|-3.281931750|j-20| \\
& +.212614100|j-21| \\
\phi_{3}(j)= & -151.9700389+26.27953346 j-11.00923102|j-2| \\
& +12.26004248|j-3|-11.76294895|j-4|+7.703347200|j-5| \\
& -3.858407670|j-6|+2.822258500|j-7|-6.554853950|j-8| \\
& -2.597214450|j-9|+.3558561000|j-13|+.7561245000|j-14| \\
& -.6907184500|j-15|+4.309898950|j-16|+8.551364500|j-17| \\
& +.212614000|j-21| . \\
\phi_{x}(j)= & 16.80925490+15.29487567 j-11.97304816|j-2| \\
& +2.865759675|j-3|+13.90385563|j-4|-15.37389633|j-5| \\
& -.230147000|j-6|-1.236058010|j-7|+5.082084830|j-8| \\
& -4.291810750|j-9|-1.363155500|j-10|+1.234058950|j-11| \\
& +3.452183650|j-12|-3.376846600|j-13|+.580606850|j-14| \\
& +4.050912850|j-15|-5.098391450|j-16|+1.294871050|j-17| \\
& -1.133544550|j-18|+4.743926900|j-19|-3.281931600|j-20| \\
& +10
\end{aligned}
$$

Finally, the similarity between $\phi_{x}(j)$ and one of the reference functions $\left(\phi_{1}(j), \phi_{2}(j)\right.$ and $\left.\phi_{3}(j)\right)$ is determined by considering Condition (10) and Eq. (11). The graphs of functions $\phi_{1}(j), \phi_{2}(j), \phi_{3}(j)$ and $\phi_{x}(j)$ are shown in figure 3 .

As can be noted from this figure, the graphs of $\phi_{2}(j)$ and $\phi_{x}(j)$ practically overlap, which implies a match between the polygonal curves $\mathbf{C}_{2}$ and $\mathbf{C}_{x}$. The difference between $\phi_{x}(j)$ and the other functions, expressed as $\left|\phi_{i}(j)-\phi_{x}(j)\right|$ for $i=1, \ldots, 3$, can be observed in figure 4 , where the coincidence between $\mathbf{C}_{2}$ and $\mathbf{C}_{x}$ can be again appreciated graphically. Moreover, in accordance with Eq. (11), a numerical value of $\epsilon=4.323412698 \times 10^{-7}$ is obtained between $\phi_{x}(j)$ and $\phi_{2}(j)$, which is in contrast with the 


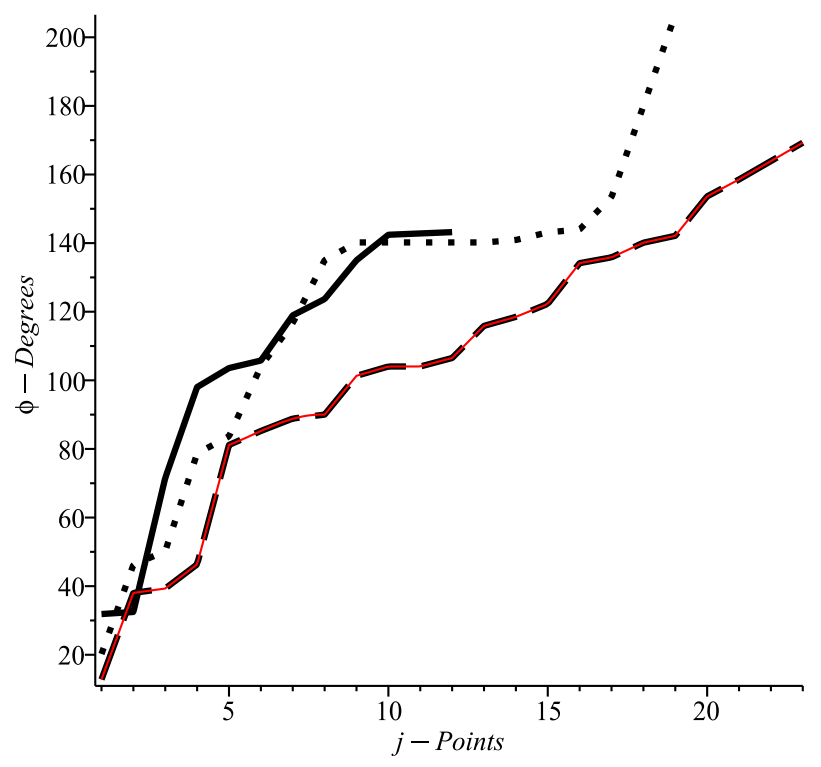

Figure 3. Graphs of functions: $\phi_{1}(j)$ (bird, solid line), $\phi_{2}(j)$ (rabbit, dashed line), $\phi_{3}(j)$ (cat, dotted line) and $\phi_{x}(j)$ (unknown, red line).

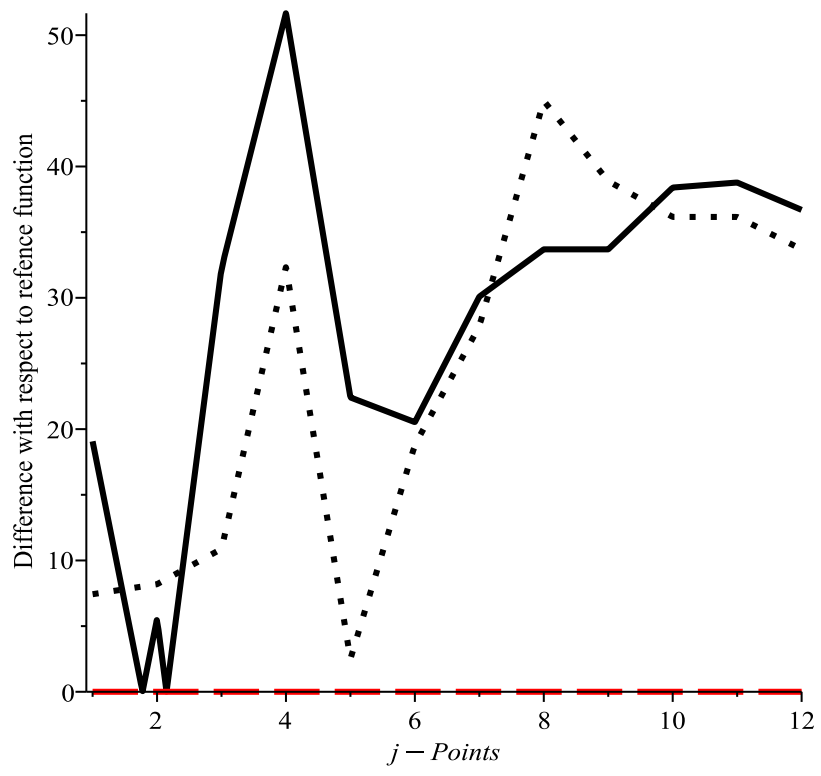

Figure 4. Difference between $\phi_{x}(j)$ and the reference functions for $\phi_{1}(j)$ (solid line), $\phi_{2}(j)$ (red, dashed line) and $\phi_{3}(j)$ (dotted line).

values of $\epsilon=325.9264155$ and $\epsilon=277.3342205$ computed by considering $\phi_{x}(j)$ with $\phi_{1}(j)$ and $\phi_{3}(j)$, respectively.

\section{Similar shape identification}

The case where the shape of the polygonal curve to be identified does not exactly correspond with one of the previously stored ones is a problem that has been approached by computational strategies more directly related to neural networks [14-19]. From the perspective of our analysis, this case imposes an important challenge to be faced: on one hand, sorting the set of angles makes it possible to establish a reference starting point in the curve, but on the other hand, in the representation of sorted angles, the graphs of piecewise-linear functions have the generalized tendency of increasing behaviour, which unfortunately limits the identification capability. In order to overcome this problem, firstly, we propose using the angle-based representation but keeping the order of appearance of the sequence of points along the polygonal curve, and secondly, with the aim of having a reference point, in this case we consider open polygonal curves.

\subsection{Illustrative application example}

In this example, we considered six images of hand shapes that correspond with the letters $\mathrm{L}, \mathrm{W}$ and $\mathrm{Y}$ of the dictionary of Mexican sign language. These letters were performed by two different test persons, here denoted as SA and SE. The signs were denoted as SAL, SAW, SAY, SEL, SEW and SEY. In accordance with our experiment, an unclassified hand shape SDX was given by a third test person, SD. With this, our main interest was focused on determining the similarity between SDX and the signs provided by SA and SE. Figure 5 shows the three test signs performed by SA and figure 6 depicts their corresponding polygonal curves obtained using a homemade program written in Matlab. In the same way, figures 7 and 8 show

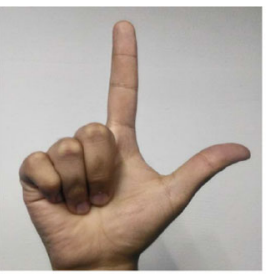

(a)

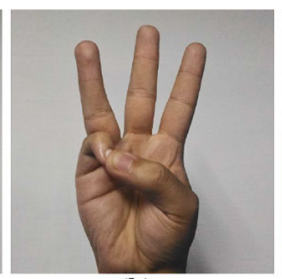

(b)

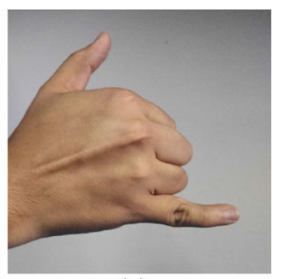

(c)
Figure 5. In accordance with the dictionary of Mexican sign language, hand shapes performed by SA: (a) the letter L, (b) the letter $\mathrm{W}$ and (c) the letter $\mathrm{Y}$.

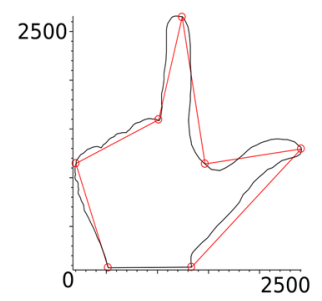

(a)

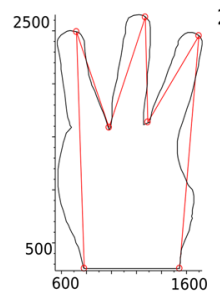

(b)

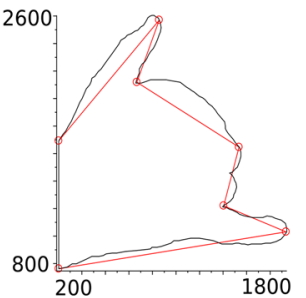

(c)
Figure 6. Polygonal curves derived from the hand shapes of SA: (a) curve $C_{S A L}$, (b) curve $C_{S A W}$ and (c) curve $C_{S A Y}$. 


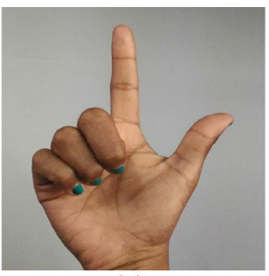

(a)

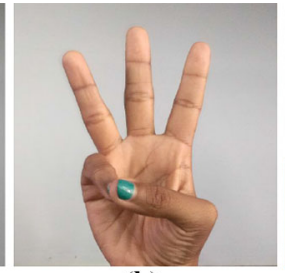

(b)

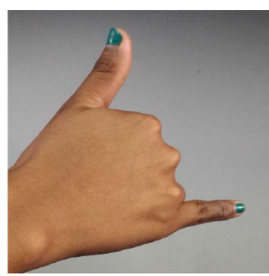

(c)
Figure 7. In accordance with the dictionary of Mexican sign language, hand shapes performed by SE: (a) the letter L, (b) the letter $\mathrm{W}$ and (c) the letter $\mathrm{Y}$.

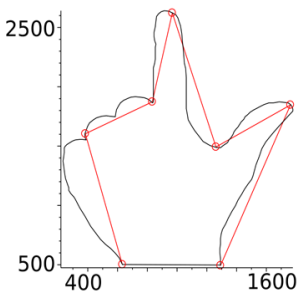

(a)

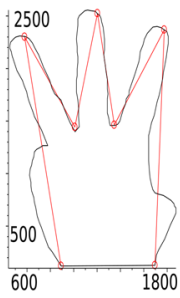

(b)

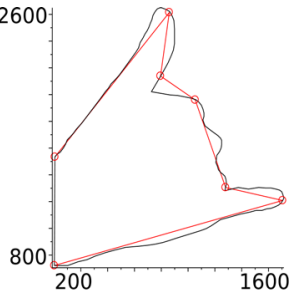

(c)
Figure 8. Polygonal curves derived from the hand shapes of SE: (a) curve $C_{S E L}$, (b) curve $C_{S E W}$ and (c) curve $C_{S E Y}$.

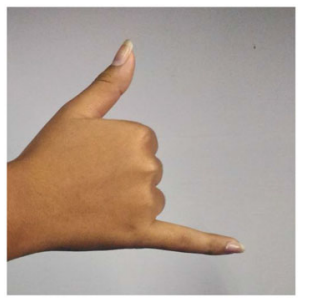

(a)

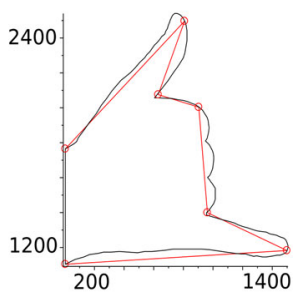

(b)
Figure 9. Non-classified sign performed by SD: (a) hand shape image and (b) polygonal curve $C_{S D X}$.

the signs and the polygonal curves corresponding to SE, whereas the image of the unknown sign and the curve SDX are depicted in figure 9.

As in the previous example, our comparative analysis was based on the premise that all the hand signs can be modelled by polygonal curves; however, in this case, remarkable differences can be distinguished. First of all, while in the previous example a complete match with the unclassified curve could be observed, in this case, only an estimation of a certain degree of similarity can be achieved because none of the considered shapes are completely equal. Next, with the purpose of defining a reference starting point, the polygonal curves considered here are open. Finally, in order to have more distinctive elements to identify the similarities among the test curves, in this case study, the piecewise-linear functions have been constructed by considering the sequence of slopes as they appear along the polygonal curve, that is, without any sorting. The set of
Table 1. Set of angles as they appear in the polygonal curve.

\begin{tabular}{lccccc}
\hline $\begin{array}{l}\text { Hand } \\
\text { shape }\end{array}$ & $\theta_{1}$ & $\theta_{2}$ & $\theta_{3}$ & $\theta_{4}$ & $\theta_{5}$ \\
\hline$C_{S A L}$ & 39.2037 & 89.1672 & 20.8283 & 131.410 & 102.573 \\
$C_{S A W}$ & 22.6060 & 27.7786 & 16.7387 & 31.6606 & 14.4887 \\
$C_{S A Y}$ & 27.6202 & 92.7520 & 101.566 & 95.7368 & 21.4067 \\
$C_{S E L}$ & 35.5495 & 69.2678 & 24.7480 & 131.061 & 108.162 \\
$C_{S E W}$ & 23.2433 & 33.0081 & 17.8880 & 36.7829 & 18.4735 \\
$C_{S E Y}$ & 28.8406 & 121.986 & 143.731 & 116.818 & 30.4634 \\
$C_{S D X}$ & 25.0104 & 117.475 & 110.201 & 81.8649 & 24.8683 \\
\hline
\end{tabular}

angles in the order in which they appear in each polygonal curve is reported in table 1.

From these data, our main interest now is to determine an appropriate model of function that best characterizes each hand shape. In this sense, for each row of the table, a function is also constructed in the form of the canonical piecewise-linear Chua-Kang model as follows: ${ }^{4}$

$$
f(j)=\alpha+\beta j+\gamma_{1}|j-2|+\gamma_{2}|j-3|
$$

where the parameters $\alpha, \beta, \gamma_{1}$ and $\gamma_{2}$ are computed as follows:

$$
\beta=\frac{J_{1}+J_{3}}{2}, \gamma_{1}=\frac{J_{2}-J_{1}}{2}, \gamma_{2}=\frac{J_{3}-J_{2}}{2},
$$

$\alpha=f(0)-2 \gamma_{1}-3 \gamma_{2}$ and $f(0)=2 m_{1}-m_{2}$.

However, as can be clearly seen in Eq. (17), the function parameters depend on two secondary variables $(J$ and $m$ ). In order to numerically compute these variables, we firstly consider the set $\left\{m_{1}, m_{2}, m_{3}, m_{4}\right\}$ (in accordance with table 1 , the number of angles minus one), with $m_{i}$, for $i=1, \ldots, 4$, defined as the angular difference $\left(m_{i}=\theta_{i+1}-\theta_{i}\right)$. Then, from these data we generate the set of points needed to construct the function in the form $\left\{\left(1, m_{1}\right),\left(2, m_{2}\right),\left(3, m_{3}\right),\left(4, m_{4}\right)\right\}$. After this, the slopes $J_{i}=m_{i+1}-m_{i}$, for $i=1, \ldots, 3$ are computed. Finally, once these variables have been determined, Eq. (17) is computed and substituted into Eq. (16).

With the aim of clarifying this construction strategy, the case of $C_{S A L}$ is considered. Applying the mentioned numerical procedure results in

\footnotetext{
${ }^{4}$ This expression is valid only for the case in which the number of points to be fit by the canonical piecewise-linear model is equal to five. The generalized form of this model is expressed as $f(j)=$ $\alpha+\beta j+\sum_{k=1}^{n} \gamma_{k}|j-k+1|$ with $(n+1)$ representing the number of points.
} 


$$
\begin{aligned}
& m_{1}=89.1672-39.2037=49.9635 \\
& m_{2}=20.8283-89.1672=-68.3389 \\
& m_{3}=131.410-20.8283=110.5817 \\
& m_{4}=102.573-131.410=-28.837
\end{aligned}
$$

and

$$
\begin{gathered}
J_{1}=-68.3389-49.9635=-118.3024, \\
J_{2}=110.5817+68.3389=178.9206, \\
J_{3}=-28.837-110.5817=-139.4187
\end{gathered}
$$

With these values, we obtain

$$
\begin{aligned}
& \beta=0.5(-118.3024-139.4187)=-128.86, \\
& \gamma_{1}=0.5(178.9206+118.3024)=148.612, \\
& \gamma_{2}=0.5(-139.4187-178.9206)=-159.17 \\
& \alpha=2(49.964)+68.339-2(148.612)+3(159.17)=348.552 .
\end{aligned}
$$

Finally, after substituting these function parameters in Eq. (16) we obtain

$$
f_{S A L}(j)=348.552-128.860 j+148.612|j-2|-159.170|j-3| .
$$

Following the same procedure, we can also find

$$
\begin{aligned}
f_{S A W}(j) & =66.2941-24.1532 j+21.0872|j-2|-29.0278|j-3|, \\
f_{S A Y}(j) & =160.562-62.4093 j+20.8373|j-2|-26.9288|j-3|, \\
f_{S E L}(j) & =302.950-103.725 j+114.536|j-2|-140.022|j-3|, \\
f_{S E W}(j) & =82.5789-31.0446 j+29.4499|j-2|-35.6097|j-3|, \\
f_{S E Y}(j) & =157.979-65.4210 j+11.3712|j-2|-5.3918|j-3|, \\
f_{S D X}(j) & =124.924-64.1995 j+39.3383|j-2|-3.7992|j-3| .
\end{aligned}
$$

Figure 10 shows graphs of these functions. The solid line represents the curves of SA and the dashed line represents the curves of SE.

In order to avoid confusion between the curves, three different colours (red, blue and green) have been chosen to easily identify each type of sign. In contrast, the black solid line depicts the graph of the unclassified sign. From this figure, the closeness between graphs also denotes the similitude between the same type of signs. For example, in this case the graphical proximity of SDX to SAY or SEY is remarkable, which allows us to conclude that SDX corresponds with the hand shape that represents the letter Y. This conclusion can also be numerically supported in accordance with the criteria considered in Eq. (11), by which the following results are obtained:

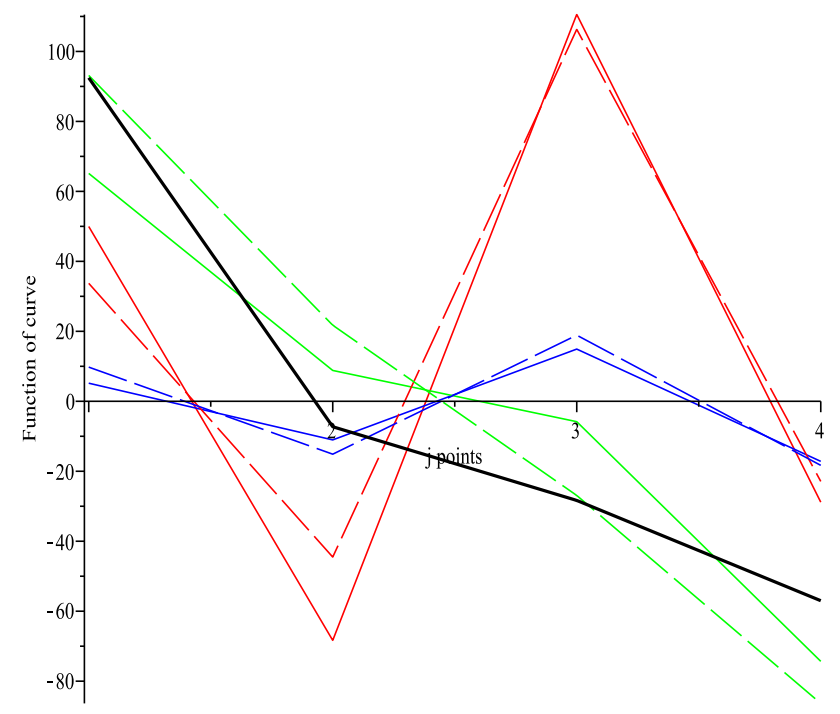

Figure 10. Graphs of functions $f_{S A L}(j)$ (red solid), $f_{S A W}(j)$ (blue solid), $f_{S A Y}(j)$ (green solid), $f_{S E L}(j)$ (red dashed), $f_{S E W}(j)$ (blue dashed), $f_{S E Y}(j)$ (green dashed) and $f_{S D X}(j)$ (black solid).

$$
\begin{aligned}
\epsilon_{S A L} & =\int_{1}^{4}\left|f_{S A L}(j)-f_{S D X}(j)\right| d j=192.898, \\
\epsilon_{S A W} & =\int_{1}^{4}\left|f_{S A W}(j)-f_{S D X}(j)\right| d j=107.118, \\
\epsilon_{S A Y} & =\int_{1}^{4}\left|f_{S A Y}(j)-f_{S D X}(j)\right| d j=41.0094, \\
\epsilon_{S E L} & =\int_{1}^{4}\left|f_{S E L}(j)-f_{S D X}(j)\right| d j=189.141, \\
\epsilon_{S E W} & =\int_{1}^{4}\left|f_{S E W}(j)-f_{S D X}(j)\right| d j=109.042, \\
\epsilon_{S E Y} & =\int_{1}^{4}\left|f_{S E Y}(j)-f_{S D X}(j)\right| d j=44.1047 .
\end{aligned}
$$

From these results, it must be noted that $\epsilon_{S A Y}$ and $\epsilon_{S E Y}$ are the values with lower deviations (41.0094 and 44.1047). This result allows us to verify that the originally unclassified sign SDX corresponds to the letter Y. Additionally, in figure 11 we provide a graphic visualization of functions:

$$
\begin{aligned}
& \left|f_{S A W}(j)-f_{S D X}(j)\right|,\left|f_{S A Y}(j)-f_{S D X}(j)\right|, \\
& \left|f_{S A L}(j)-f_{S D X}(j)\right|,\left|f_{S E L}(j)-f_{S D X}(j)\right|, \\
& \left|f_{S E W}(j)-f_{S D X}(j)\right| \quad \text { and }\left|\mathrm{f}_{S E Y}(\mathrm{j})-\mathrm{f}_{\mathrm{SDX}}(\mathrm{j})\right| .
\end{aligned}
$$

From this figure, it can be observed that the graph of $\left|f_{S A Y}(j)-f_{S D X}(j)\right|$ and the graph of $\left|f_{S E Y}(j)-f_{S D X}(j)\right|$ (green) have lower levels, which definitely represents another way to confirm the close correspondence between SDX and the letter Y. 


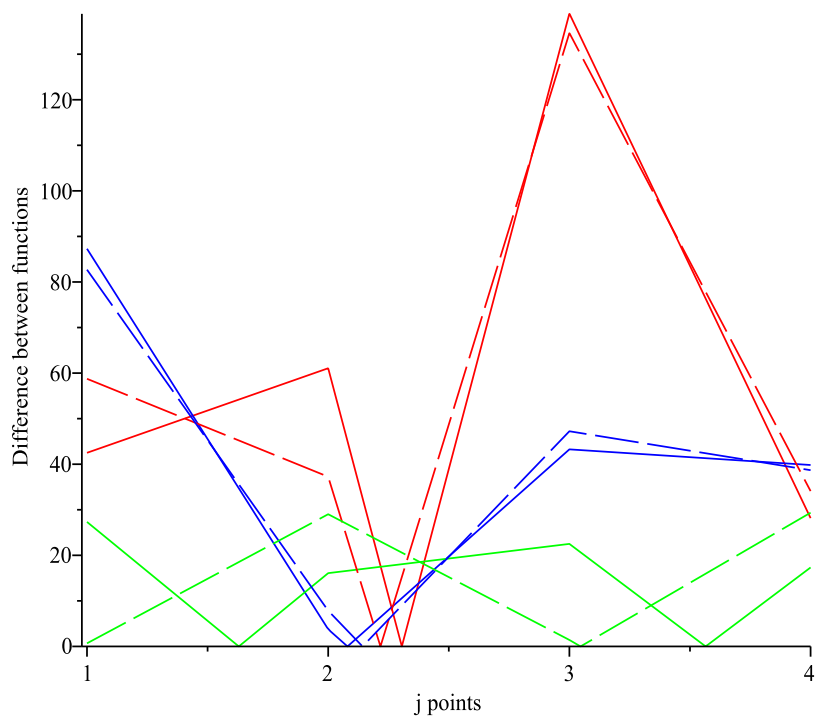

Figure 11. Difference among the reference function $f_{S D X}(j)$ and the set of functions $\left\{f_{S A L}(j), f_{S A W}(j), f_{S A Y}(j), f_{S E L}(j), f_{S E W}(j)\right.$, $\left.f_{S E Y}(j)\right\}$. With respect to $f_{S A L}(j)$ (red solid), with respect to $f_{S A W}(j)$ (blue solid), with respect to $f_{S A Y}(j)$ (green solid), with respect to $f_{S E L}(j)$ (red dashed), with respect to $f_{S E W}(j)$ (blue dashed), with respect to $f_{S E Y}(j)$ (green dashed).

\section{Comparative analysis and validation}

In order to validate the performance of the proposed description in shape identification, a comparative analysis with other two representative methods based on polygonal representation is provided. The proposal based on piecewise linearity was compared to the Fourier descriptors [20-23] and the canonical shape representation [24] methods. The comparison was performed considering the example of hand shape recognition described in the previous section. The same set of polygonal curves (figures 6,8 and $9 \mathrm{~b}$ ) were processed in accordance with the particular requirements of each method. For instance, in the Fourier descriptors, the centroid computation, the construction of a mesh distance vector (distances measured from centroid to breakpoints) and the application of Fourier discrete transform were considered. In the canonical shape representation, in addition to calculating centroid and performing Fourier discrete transform, sorting and interpolation of data procedures were also performed; moreover the construction of a peculiar distance vector (distances measured from centroid to breakpoints as well as distances between consecutive breakpoints) was also included. In contrast, in the piecewise-linear proposal the only requirement was the angles computation and the function construction. In table 2, the results obtained in the three analysed methods by applying the same identification criteria of (10) are summarized.

The first aspect that can be highlighted is the higher spread in the values of error that can be achieved by the proposal. This fact represents an advantage over the other methods because in accordance with (10), the higher the dispersion in error, the more effective the recognition. That is, the result of comparing polygonal curves with a completely different shape has a high value of error. On the contrary, the comparison of similar shapes produces a low error. Therefore, a set with dispersed values of error allows differentiation of the shape of each curve more easily. This characteristic is more clearly portrayed in figure 12 , which shows the normalized-average error (bar graphs) and the standard deviation (vertical lines) derived from the results of table 2. From this figure, it can be clearly seen that higher error and deviation can be found in the proposal.

In order to compare the computational performance of the three methods, the execution time required to construct the polygonal description and the particular identification procedure was calculated for each method with the aid of Maple v15 in a Workstation Intel Xeon $2.67 \mathrm{GHz}$ CPU X5550. In figure 13, a comparative analysis for run-time among the three identification methods is shown.

An important aspect that can be observed from this figure is the lowest run-time required in the proposal (60.640, 7.812 and $2.860 \mathrm{~s}$, for the canonical form, Fourier descriptors and the proposal, respectively). The main reason for this is the fact that the proposal excludes not only the centroid calculation but also the construction of distance vectors, which implies saving of time that substantially speeds up the computation.

Table 2. Identification results by applying the criteria of (10).

\begin{tabular}{|c|c|c|c|c|c|c|}
\hline \multirow[b]{2}{*}{ Curve } & \multicolumn{2}{|c|}{ Proposal } & \multicolumn{2}{|c|}{ Fourier descriptors } & \multicolumn{2}{|c|}{ Canonical form } \\
\hline & SA & SE & SA & SE & SA & SE \\
\hline $\mathrm{L}$ & 192.89 & 189.14 & 3790.10 & 2309.50 & 8129.12 & 6162.92 \\
\hline W & 107.11 & 109.04 & 7269.53 & 7332.43 & $17,762.32$ & $17,099.53$ \\
\hline$Y$ & 41.10 & 44.10 & 3527.01 & 2944.21 & 5056.79 & 4090.25 \\
\hline
\end{tabular}




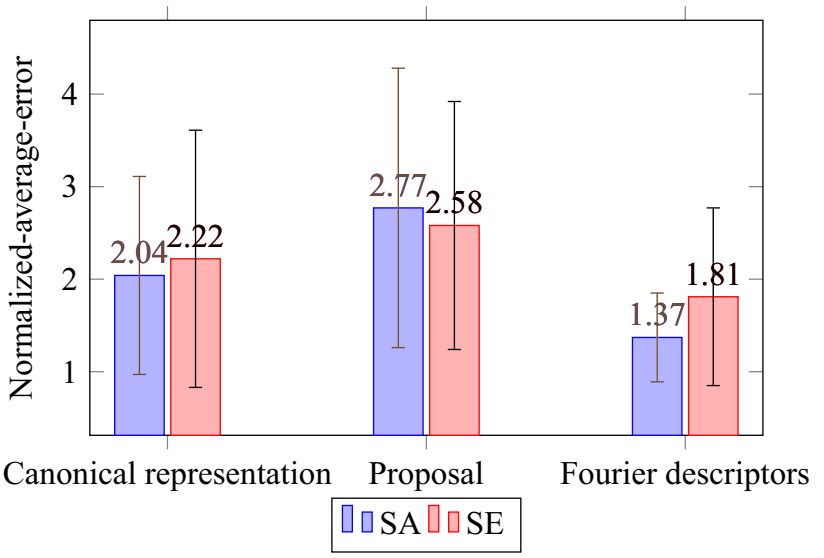

Figure 12. Identification results: normalized-average-error and standard deviation.

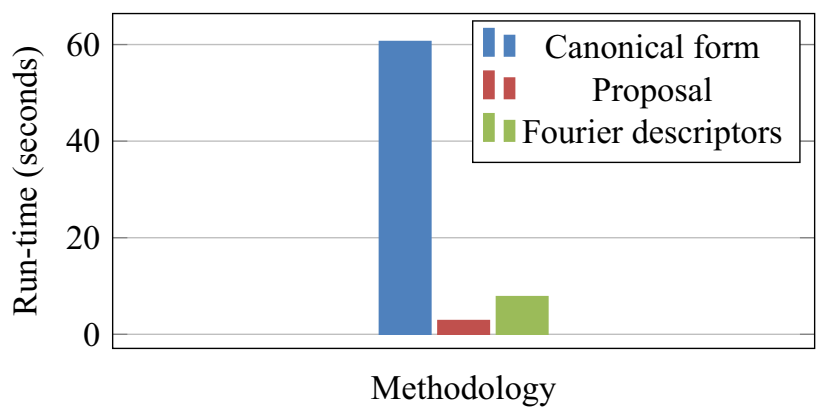

Figure 13. Run-time comparative among the three identification methods.

\section{Conclusions}

A new representation for polygonal curves with potential application in shape identification was presented. Specifically, the problem of identifying the shape of a given polygonal curve from a set of previously stored ones was approached. In our proposal, two case studies were considered: the first one dealt with the problem of identifying a polygonal curve whose shape had a perfect matching correspondence with the shape of a curve stored in a data set, and the second one covered the problem of identifying polygonal curves with similar shapes. In both cases, the angle-based representation was expressed in the form of the canonical piecewise-linear model. However, in the case of the identification of two equal shapes, a sorting procedure was an essential component, while in the similar shape case, it was not considered. Moreover, it is also important to remark that in the first case study the principle of similarity geometry that allows a polygon to be recognized independently of scale, translation and rotation was exploited. Finally, it must be mentioned that through illustrative examples, the effectiveness of the proposed representation applied in shape identification was verified under the two mentioned scenarios.

\section{References}

[1] Alt H, Behrends B and Blömer J 1995 Approximate matching of polygonal shapes. Ann. Math. Artif. Intell. 13: 251-265

[2] Kashyap R L and Oommen B J 1982 A geometrical approach to polygonal dissimilarity and shape matching. IEEE Trans. Pattern Anal. Mach. Intell. 4: 649-654

[3] Eckhardt U and Reiter H 2004 Polygonal representations of digital sets. Algorithmica 38: 5-23

[4] Hosur P I and Ma K K 1999 A novel scheme for progressive polygon approximation of shape contours. In: Proceedings of the Third IEEE Workshop on Multimedia Signal Processing, pp. 309-314

[5] Guanghui L and Chuanbo C 2007 A new approach for polygonal approximation of shape contours using genetic algorithm. In: Proceedings of the 2nd IEEE Conference on Industrial Electronics and Applications, pp. 763-768

[6] Tan W, Zhao S, Wu C and Li C 2008 A novel approach to 2-D shape representation based on equilateral polygonal approximation. In: Proceedings of the International Conference on Computer Science and Sofware Engineering, pp. 1021-1024

[7] Kaup A and Heuer J 2000 Polygonal shape descriptors - an efficient solution for image retrieval and object localization. In: Proceedings of the Conference Record of the ThirtyFourth Asilomar Conference on Signals, Systems and Computers, pp. 59-64

[8] Gheibi A, Davoodi M, Javad A, Panahi F, Aghdam M M, Asgaripour M and Mohades A 2011 Polygonal shape reconstruction in the plane. IET Comput. Vis. 5: 97-106

[9] Gomes J, Velho L and Costa Sousa M 2012 Computer graphics: theory and practice. New York: AK Peters/CRC Press, pp. 9-14

[10] Chua L O and Kang S M 1977 Section-wise piecewise-linear functions: canonical representation, properties and applications. Proc. IEEE 65: 915-929

[11] Kang S M and Chua L O 1978 A global representation of multidimensional piecewise-linear functions with linear partitions. IEEE Trans. Circuits Syst. 25: 938-940

[12] Chua L O and Deng A C 1986 Canonical piecewise-linear modeling. IEEE Trans. Circuits Syst. 33: 511-525

[13] Chua L O and Deng A C 1988 Canonical piecewise-linear representation. IEEE Trans. Circuits Syst. 35: 101-111

[14] El-Alfy E S M and Abdel-Aal R E 2014 Abductive learning ensembles for hand shape identification. Cogn. Comput. 6: 321-330

[15] Tralic D, Bozek J and Grgic S 2011 Shape analysis and classification of masses in mammographic images using neural networks. In: Proceedings of the 18th International Conference on Systems, Signals and Image Processing, pp. 1-5

[16] Zhang X L, Zhao L, Zhao W B and Xu T 2015 Novel method of flatness pattern recognition via cloud neural network. Soft Comput. 19: 2837-2843

[17] Smetanin Y G 1998 Neural networks as systems for recognizing patterns. J. Math. Sci. 89: 1406-1457

[18] Lin S W, Chou S Y and Chen S C 2007 Irregular shapes classification by back-propagation neural networks. Int. J. Adv. Manuf. Technol. 34: 1164-1172 
[19] Datta A and Parui S K 1998 Shape extraction: a comparative study between neural network-based and conventional techniques. Neural Comput. Appl. 7: 343-355

[20] Dalitz C, Brandt C, Goebbels S and Kolanus D 2013 Fourier descriptors for broken shapes. EURASIP J. Adv. Sig. Process. 2013: 1-11

[21] Waldchen J and Mader P 2018 Plant species identification using computer vision techniques: asystematic literature review. Arch. Comput. Methods Eng. 25: 507-543
[22] Ganpatrao N G and Ghosh J K 2014 Information extraction from topographic map using colour and shape analysis. Sadhana 39: 1095-1117

[23] Manipoonchelvi P and Muneeswaran K 2014 Multi region based image retrieval system. Sadhana 39: 333344

[24] Soysal O M and Chen J 2012 Object recognition by spectral feature derived from canonical shape representation. Mach. Vision Appl. 24: 855-868 